\title{
Loss of FVIII Expression by Possible Interlinked Immune and Cellular Stress Response in Hemophilia A Mice Treated with AAV Gene Therapy
}

\author{
Chris Rogers ${ }^{1}$, Kentaro Yamada ${ }^{1}$, Thais Bertolini ${ }^{1}$, Roland Herzog ${ }^{1}$ \\ 1. Herman B Wells Center for Pediatric Research, IU School of Medicine
}

\section{Background}

Hemophilia $\mathrm{A}$ is an X-linked genetic disorder caused by a mutation in the gene for factor VIII (FVIII) protein that reduces the ability of blood to clot. Clinical drug trials have shown the potential of adenoassociated virus (AAV) gene therapy as a one-time treatment for hemophilia A that can produce sustained high levels of FVIII. However, a gradual decline in protein levels has been observed in patients after 2-4 years. The hypothesis being tested in the Herzog Lab is that an interlinked immune and cellular stress response could be causing the loss of expression.

\section{Methods}

Two groups of Hemophilia A mice were administered AAV therapy, with one group receiving recurrent doses of Rapamycin. Blood samples were taken at weeks 4, 8, 12 and 14. Mice were euthanized at weeks 4,8 , and 14, and their livers were harvested. qPCR was used to measure AAV copy numbers and FVIII mRNA at 4, 8, and 14 weeks. Cryosections of mice livers from weeks 4, 8, and 14 were stained with antibodies for FVIII protein and CD8.

\section{Results}

qPCR showed roughly half as much AAV copy numbers in the rapamycin group at all time points, and little difference in FVIII mRNA between the groups. There was also a large decrease in AAV copy numbers and FVIII mRNA in both groups between 8 and 14 weeks. Immunohistochemistry showed less CD8 and more FVIII signal in mice treated with rapamycin.

\section{Discussion}

Experiments are currently being performed to investigate the decline in AAV copy numbers and mRNA between weeks 8 and 14. The immunohistochemistry data shows a relationship between increased FVIII protein levels and decreased cellular immune response but does not explain the gradual decline in FVIII. Further investigation into FVIII expression following AAV gene therapy could lead to an effective onetime treatment for hemophilia A. 